\title{
Motor-evoked potential monitoring for intramedullary spinal cord tumor surgery: correlation of clinical and neurophysiological data in a series of 100 consecutive procedures
}

\author{
Karl F. Kothbauer, M.D., Vedran Deletis, M.D., Ph.D., and Fred J. Epstein, M.D. \\ Institute of Neurology and Neurosurgery, Beth Israel Medical Center North Division, New York, New \\ York
}

Resection of intramedullary spinal cord tumors carries a high risk for surgical damage to the motor pathways. This surgery is therefore optimal for testing the performance of intraoperative motor evoked potential (MEP) monitoring. This report attempts to provide evidence for the accurate representation of patients' pre- and postoperative motor status by combined epidural and muscle MEP monitoring during intramedullary surgery.

The authors used transcranial electrical motor cortex stimulation to elicit MEPs, which were recorded from the spinal cord (with an epidural electrode) and from limb target muscles (thenar, anterior tibial) with needle electrodes. The amplitude of the epidural MEPs and the presence or absence of muscle MEPs were the parameters for MEP interpretation. A retrospective analysis was performed on data from the resection of 100 consecutive intramedullary tumors and MEP data were compared with the pre- and postoperative motor status.

Intraoperative monitoring was feasible in all patients without severe preoperative motor deficits. Preoperatively paraplegic patients had no recordable MEPs. The sensitivity of muscle MEPs to detect postoperative motor deficits was $100 \%$ and its specificity was $91 \%$. There was no instance in which a patient with stable MEPs developed a motor deficit postoperatively. Intraoperative MEPs adequately represented the motor status of patients undergoing surgery for intramedullary tumors. Because deterioration of the motor status was transient in all cases, it can be considered that impairment of the functional integrity of the motor pathways was detected before permanent deficits occurred.

Key Words * spinal cord * intramedullary tumor * intraoperative monitoring * motor evoked potentials * neurophysiology

The primary objective of intraoperative neurophysiological monitoring during neurosurgery is to avoid permanent neurological injury resulting from surgical manipulation. To achieve this objective, monitoring must be practical, safe, based on a sound neurophysiological concept, correlate with the clinical findings, and warn the surgeon before a permanent injury occurs. Eventually the use of such a technique should be shown to improve the overall outcome of patient treatment. 
During surgery of intramedullary spinal cord tumors, a neurophysiological monitoring technique is most rigorously tested for its performance because this surgery is considered to be "high risk" in terms of direct and selective surgical damage to the functional integrity of the motor pathways. Both patients and surgeons, however, are generally willing to tolerate a certain degree of postoperative sensory deficits, if this is inevitable to achieve a total and safe removal of the lesion. Furthermore, the surgical approach to the tumor through the dorsal columns makes some sensory deficits almost unavoidable; however, this is in most cases the only way to approach intramedullary tumors.[11] Thus the functional integrity of the motor pathways needs to be monitored directly. The main objective of this report is to provide evidence for the correlation between the intraoperative neurophysiological data and motor status.

\section{CLINICAL MATERIAL AND METHODS}

\section{Patient Data}

Between January 1996 and January 1998, intraoperative MEP monitoring was used in 100 consecutive operations performed by the senior author (F.J.E.) in patients with intramedullary spinal cord tumors. Ninety-eight patients (median age 29 years, range 1-75 years; 54 male, 44 female) were included; two of them underwent two operations during that period. The histopathological findings are provided in Table 1. The median size of the lesions spanned four spinal segments (range one-11). Fifteen of these procedures were reoperations. The neurophysiological monitoring techniques were originally established on the basis of an institutional review board-approved protocol at New York University. Since 1994 all of the techniques presented here have been part of our neurophysiological monitoring routine for spinal cord tumors. Consent from patients was obtained together with the consent for surgery.

\begin{tabular}{|cc|}
\hline \multicolumn{3}{c}{ TABLE 1 } \\
HISTOPATHOLOGKAL FIND NGS IN 100 CONSECUTIVE SURGI- \\
CALLY TREATED INTRAMEDULLARY SPINAL CORD TUMORS \\
\hline \hline TImor Type & No. of Tumors \\
\hline ependymoma & 34 \\
pilocytic astrocytoma & 10 \\
anaplastic astrocytoma & 8 \\
astrocytoma & 6 \\
hemangiotlastoma & 6 \\
ganglioglioma & 5 \\
lipoma & 4 \\
cavernoma & 3 \\
mined glioneural tumor & 3 \\
other & 6 \\
unknown & 15 \\
\hline
\end{tabular}

\section{Neurophysiological Monitoring}

The intraoperative motor evoked potential (MEP) monitoring techniques used are the result of lengthy and ongoing endeavors by many investigators including the authors.[3,5,7,8,15,17,19,21,22,24-29,31,35] For clarity the technique will be outlined here.

Motor potentials were evoked using transcranial electrical stimulation. The stimulus points were C3, C4, $\mathrm{C} 1, \mathrm{C} 2, \mathrm{Cz}$, and a point $6 \mathrm{~cm}$ in front of $\mathrm{Cz}$ (International 10/20 EEG electrode system) (Fig. 1). Electrical stimulation was performed using rectangular constant-current stimuli of $500-\mu$ sec duration and intensities between $15 \mathrm{~mA}$ and $200 \mathrm{~mA}$. 


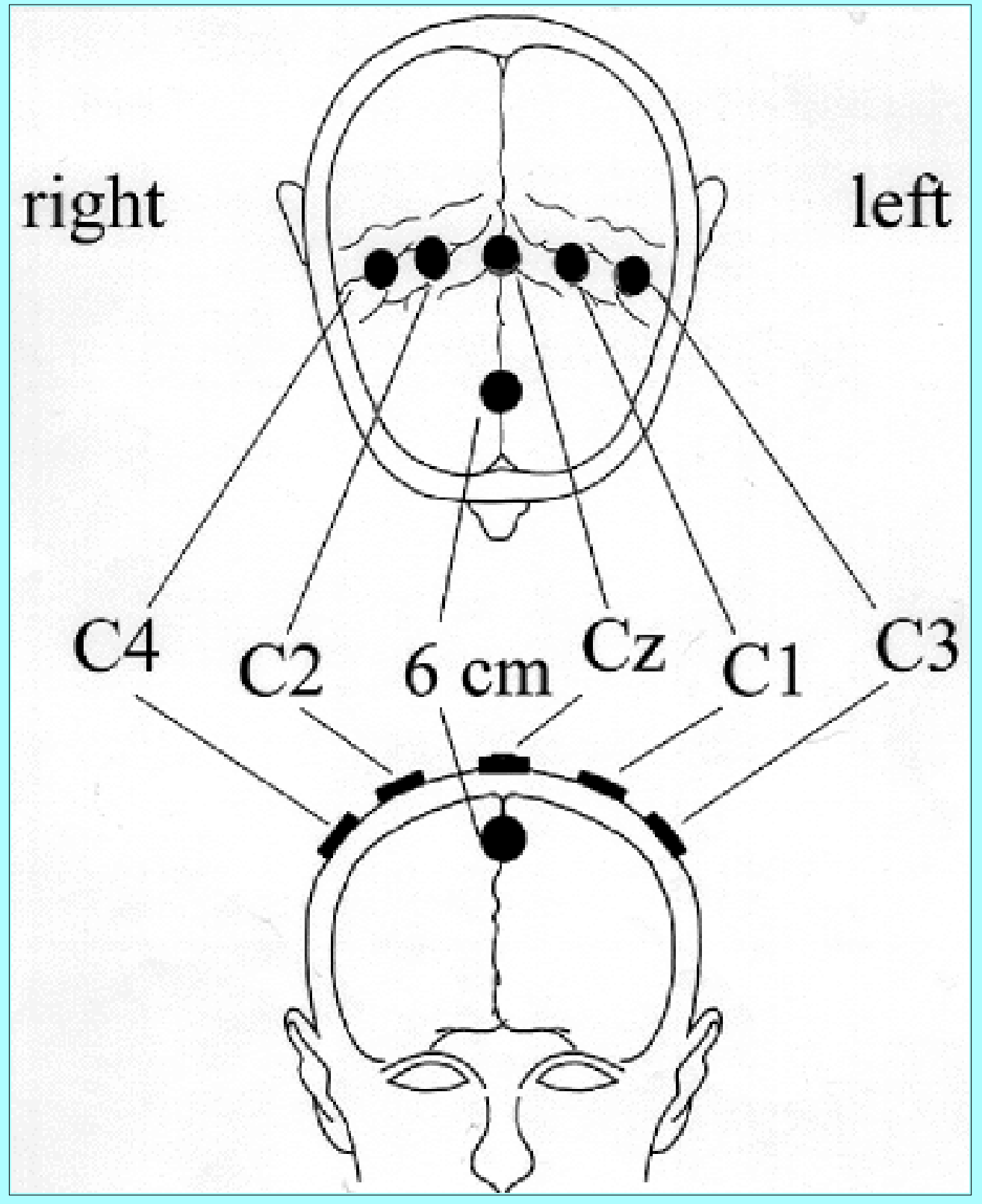

Fig. 1. Schematic drawing showing the stimulus points for transcranial electrical stimulation of the motor cortex.

Epidural MEPs (D-waves) elicited with single stimuli (single stimulus technique) (Fig. 2) were recorded from the spinal cord with an electrode (Type JX-330; Arrow International, Inc., Reading, PA) inserted into the spinal epidural space by the surgeon after laminectomy. 


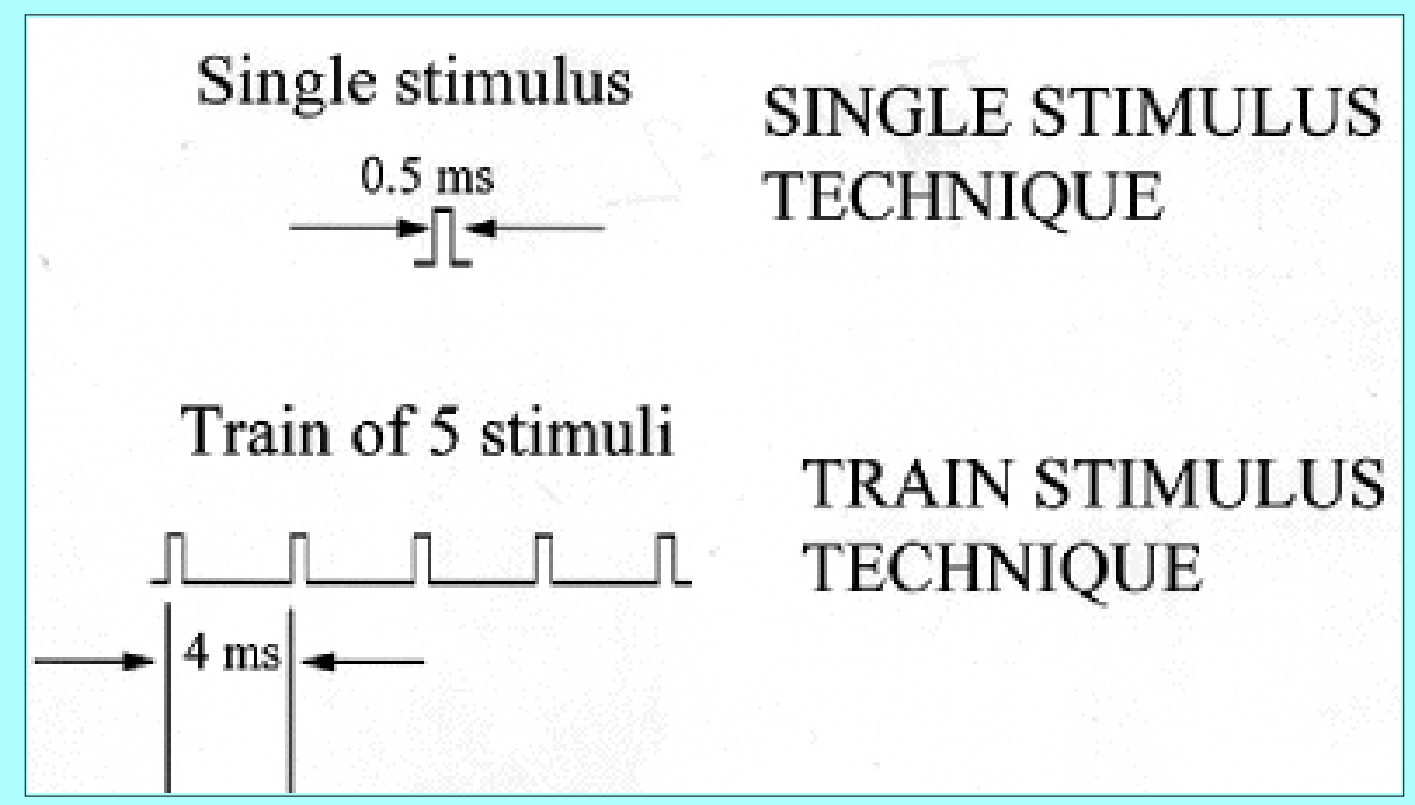

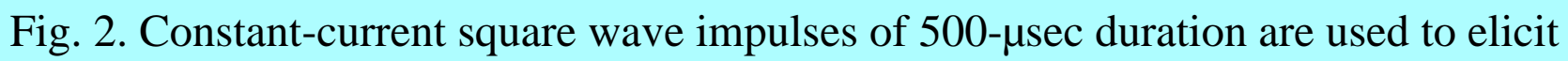
D-waves (single-stimulus technique). A short train of five such stimuli, $4 \mathrm{msec}$ apart, is used to elicit muscle MEPs (train-stimulus technique).

One electrode was placed caudally, and, if the tumor location permitted, another was placed cranially as a control (Fig. 3). The signals were amplified 10,000 times, filtered between $1.5 \mathrm{~Hz}$ and $1700 \mathrm{~Hz}$, and recorded on epochs of $20 \mathrm{msec}$. Baseline recordings were obtained before the dura was opened. The signal usually required no averaging. The stimulations were repeated at a rate of 0.5 to $2 \mathrm{~Hz}$. This repetition rate together with the feature of a single stimulus-single response pattern provided fast feedback, virtually "real-time." Recordings were continued throughout the entire surgical procedure. The parameter monitored in epidural recordings was the peak-to-peak amplitude of the D-wave. (D-wave and epidural MEPs are used synonymously in this article.) 


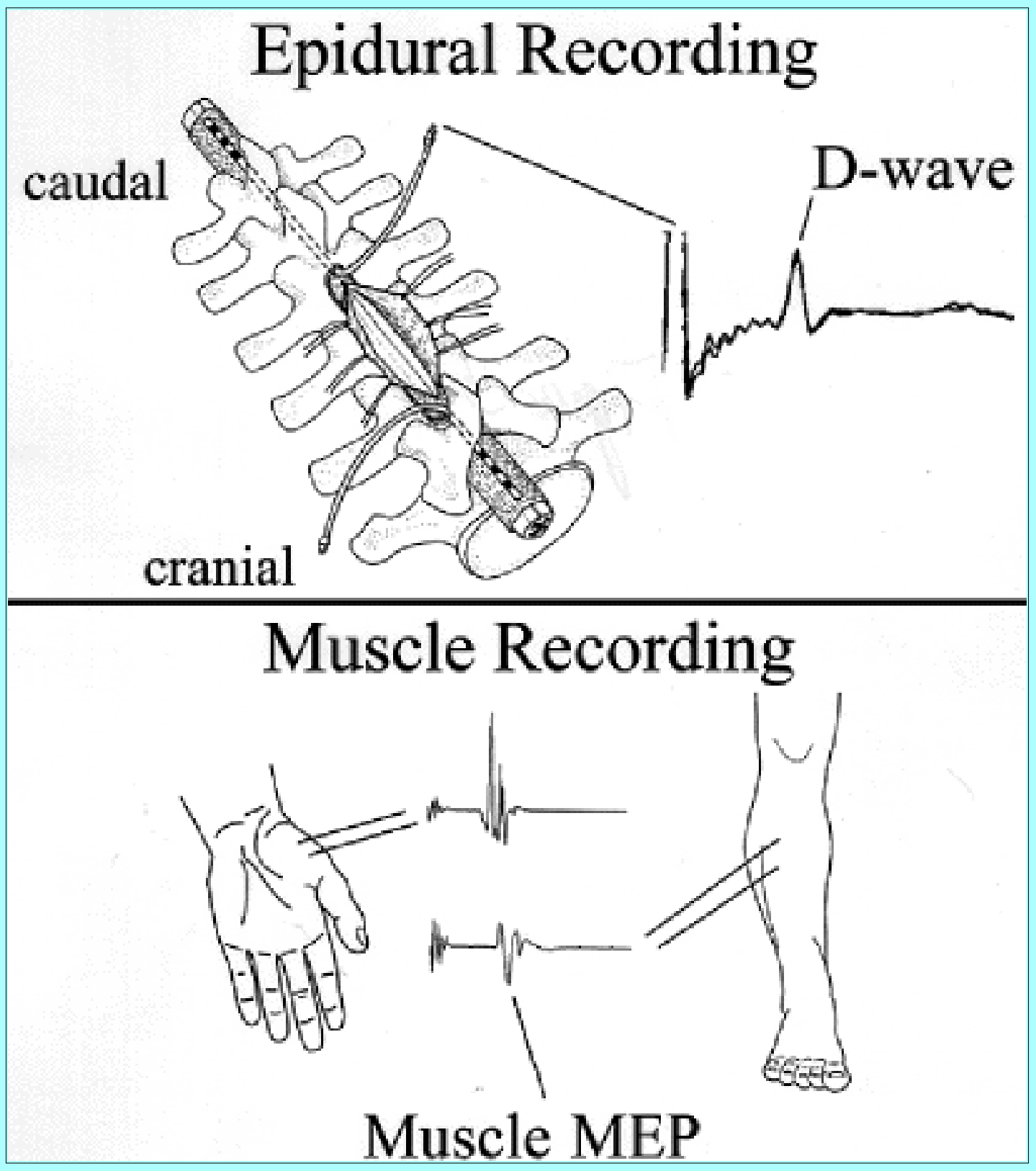

Fig. 3. Schematic drawings demonstrating the sites in which D-waves and muscle MEPs are recorded, together with actual recordings.

Muscle MEPs were elicited with a train-stimulation technique consisting of a short train of five to seven stimuli with $4 \mathrm{msec}$ interstimulus intervals (Fig. 2). Compound muscle action potentials were recorded using needle electrodes from target muscles in all four extremities, usually the thenar and anterior tibialis. The signals were amplified 10,000 times and recorded on epochs of $100 \mathrm{msec}$ with a filter bandpass from 1.5 to $853 \mathrm{~Hz}$. Baseline recordings were obtained before or shortly after skin incision. The signals also did not require averaging and could be repeated at a rate of 0.5 to $2 \mathrm{~Hz}$. Therefore, real-time feedback was provided here as well. Using the focal anode as a stimulating electrode, a montage of $\mathrm{C} 1 / 2$ or C2/1 was first attempted to elicit muscle MEPs in all four extremities. In individual cases C3/4, C4/3, or $\mathrm{Cz} / 6$ were used as alternatives. Muscle MEPs were recorded in a rapidly alternating fashion with epidural MEPs. The parameter monitored was the presence or absence of muscle MEPs in the target muscles within the stimulus-intensity constraints of 160 to a maximum of $200 \mathrm{~mA}$. All recordings were obtained using the Sentinel-4 EP analyzer (Axon Systems Inc., Hauppauge, NY) equipped with dedicated software for controlling transcranial stimulation paradigms. 


\section{Anesthesia Management}

Anesthesia management compatible with all surgical procedures requiring intraoperative MEP monitoring consisted of a constant infusion of propofol (100-150 $\mu \mathrm{g} / \mathrm{kg} / \mathrm{minute})$ and fentanyl (1 $\mu \mathrm{g} / \mathrm{kg} /$ hour), supplemented with nitrous oxide not exceeding $50 \mathrm{Vol} . \%$. Bolus injections of both intravenous agents were avoided whenever possible. Halogenated anesthetics were not used. Short-acting muscle relaxants were only administered for intubation but not thereafter.

\section{Clinical Analysis and Neurophysiological Correlation}

The clinical data were retrospectively analyzed from the patients' charts, surgical reports, and follow-up notes. Pre- and postoperative motor function was classified as normal (no focal motor deficit), slightly paretic (motor deficit not exceeding 4/5 and not entirely impairing the extremity's function, walking not impaired), severely paretic (motor deficit $3 / 4$ or worse, significantly impaired function of extremity, or inability to walk) and plegic (0/5 or $1 / 5)$. Data derived from upper-extremity monitoring in cases of tumors that only involved the cervical cord were accorded with data regarding the lower extremities. Postoperative motor function was evaluated not less than 12 hours postprocedure.

The extent of tumor removal was assessed as gross-total resection (90\% resection or more), subtotal resection $(50-90 \%)$, partial resection $(<50 \%)$, or biopsy, according to the postoperative magnetic resonance imaging. The MEP data were analyzed and categorized according to the principles outlined in Table 2.

\begin{tabular}{|ccc|}
\hline & \multicolumn{3}{c|}{ TABLE 2 } \\
& PRINC IPLES OF COMBNED MEP DATA INTER PRETATION \\
\hline \hline D-wave & Muscle MEP & Postop Motor Stat us \\
\hline unchanged & preserved & unchanged \\
unchanged & lost uni- or tilat & temporary motor de ficit \\
$30-50 \%$ decrease & preserwed & unchanged \\
$30-50 \%$ decrease & lost uni- or tilat & tem porary motor de ficit \\
$>50 \%$ decrease & lost bilat & long-term motor de ficit \\
\hline
\end{tabular}

\section{RESULTS}

\section{Correlation of Clinical Findings with Intraoperative MEPs}

Before surgery 93 of the 98 patients had normal or slightly impaired motor status. In all 93 cases, muscle MEPs could be recorded at the beginning of surgery ("baseline"). Epidural MEPs were recordable in 59 of the 86 cases not involving the conus medullaris. Eight patients had severe motor deficits or were paralyzed, and none of them had recordable epidural or muscle MEPs.

Intraoperatively the combined epidural and muscle MEP data (Table 2) indicated jeopardized functional integrity of the motor pathways in $40(43 \%)$ of the 93 patients in whom responses could be monitored. In $58(62 \%)$ of 93 the data indicated preserved functional integrity of the motor pathways.

Postoperatively a short-term motor status deterioration was noted in $35(38 \%)$ of 93 patients, but all these deficits eventually resolved and no patient experienced a permanent surgery-related severe motor deficit. In the other 58 cases $(62 \%)$ the motor status was not significantly changed after surgery.

With five false-positive and no false-negative results, the sensitivity of intraoperative MEP changes that 
heralded postoperative worsening of motor status was $100 \%$. Specificity was $91 \%$. The "false-positive" cases were those in which MEPs indicated a motor deficit but none was present after surgery. In all five of the patients, muscle MEP loss occurred: in three the D-wave did not change; in the other two the D-wave was not recordable at baseline because the patients' tumors were in the conus medullaris.

\section{Feasibility and Practicality of Monitoring}

All patients without severe motor deficits could be monitored using MEPs. The recordings were robust (between $10 \mu \mathrm{V}$ and $40 \mu \mathrm{V}$ amplitudes in epidural and up to $2 \mathrm{mV}$ in muscle MEPs). Changes due to nonsurgical influences (intravenous bolus of anesthetic, temperature or blood pressure changes) could be recognized. Bolus application of both propofol and fentanyl significantly affected muscle MEPs or completely abolished them but did not affect epidural MEPs.

Electrodes and leads were attached to the patient concurrently with anesthesia preparations (intubation, intravenous and intraarterial lines). Additional time, if any, required for routine monitoring preparation never exceeded 5 to 10 minutes. The epidural recording electrodes leading from the surgical field to a preamplifier attached to the end of the operating table were inserted by the surgeon and did not disturb the microsurgical dissection.

\section{Adverse Events}

There were no complications such as injury or infection due to electrode placement or stimulation, no spinal epidural hematomas following insertion of epidural electrodes, no neurological complications associated with transcranial motor cortex stimulation, and no seizures occurred.

The train-stimulus technique necessary to elicit muscle MEPs frequently elicited slight twitches not only in the limb target muscles but also in paraspinal or masticatory muscles. Particularly when a C3/4 stimulation is used, the temporalis muscles are directly activated; consequently minor bite injuries to the tongue occurred in two patients. (When this was recognized, further injuries were avoided by tongue protection with a padded Guedel tube.) The twitching of paraspinal muscles frequently produced slight movements. In most cases, however, this did not interfere with microsurgical dissection. In those cases in which it was too strong, epidural recordings were used continuously (the single-stimulus technique does not produce twitching), and muscle MEPs were checked at brief intervals during microsurgical dissection.

\section{Influence of MEP Data on Surgical Strategy and Extent of Resection}

In five patients the surgical report explicitly noted that the procedure was terminated due to a change in MEPs, either a loss of muscle MEPs or loss of muscle MEPs combined with decrease in D-wave amplitude (Table 2). All these patients were paraplegic after surgery but recovered in the early postoperative period. In four of them the termination of surgery resulted in only subtotal or partial tumor resection. In another four patients the initial surgical strategy was conservative because of early deterioration in MEP data or difficulty in obtaining them at all. Furthermore, in four instances stable recordings encouraged the surgeon to proceed with tumor resection, although the anatomical situation suggested otherwise; this is illustrated by the patient in Case 4. In all patients in whom epidural MEPs could not be monitored, a decidedly conservative approach was taken because these patients have an elevated risk for postoperative motor deficits.[25] In the other cases with intraoperative MEP change, it either occurred at the very end of the resection or the resection was continued and finished in the face of changes that would indicate a short-term deficit with subsequent recovery. The information regarding the 
neurophysiologically intact functional integrity of the motor pathways exerted influence in every procedure.

\section{ILLUSTRATIVE CASES}

\section{Case 1}

This 14-year-old girl presented with progressive dysesthesias in the left arm and leg and a slight weakness in the left extremities. Magnetic resonance imaging revealed an intramedullary spinal cord tumor extending from $\mathrm{C}-3$ to $\mathrm{C}-7$, which turned out to be an astrocytoma. During microsurgical gross-total resection of the lesion, monitoring of muscle MEPs in the anterior tibial muscles bilaterally showed preserved responses until the end of surgery (Fig. 4 upper). Epidural MEPs (Fig. 4 lower) showed no change in the amplitude of the D-wave. The MEP data indicated preserved functional integrity of the motor system. Postoperatively the patient's motor status was unchanged.

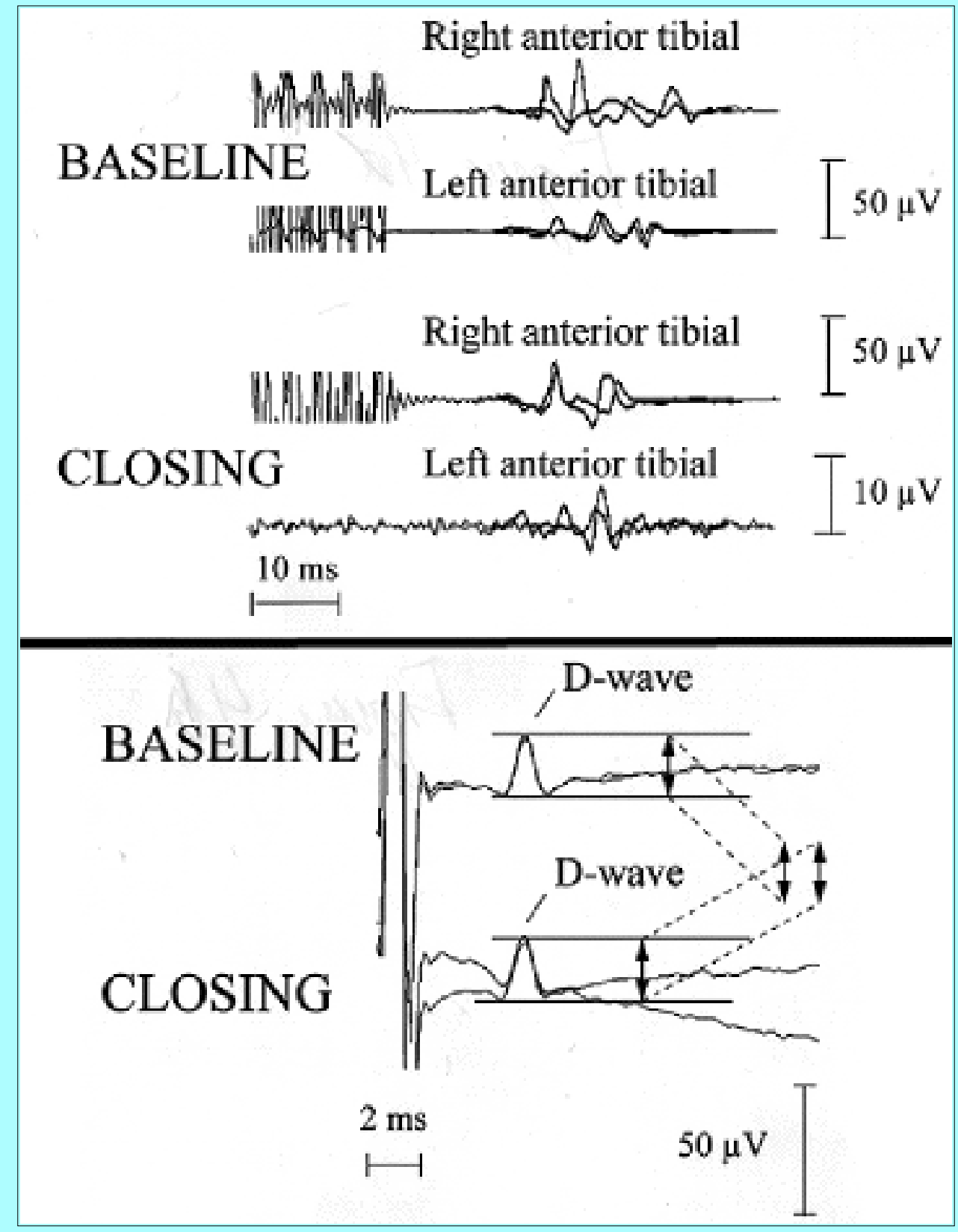

Fig. 4. Case 1. Upper: Muscle MEPs in the lower extremities evoked by a train of five stimuli with $120 \mathrm{~mA}$ at $\mathrm{C} 1 / 2$ and $\mathrm{C} 2 / 1$. Note that the amplitude in the left anterior tibial 
muscle is decreased at closing. Nevertheless the response is present. The patient had no motor deficits after surgery. Lower: The peak-to-peak amplitude of the epidurally recorded D-wave remained unchanged throughout the procedure, indicating intact functional integrity of the corticospinal tract.

\section{Case 2}

This 9-year-old girl underwent resection of a cervicothoracic intramedullary ganglioglioma. There was no preoperative motor deficit. Toward the end of the procedure, when only a small amount of tumor tissue remained, a sudden loss of muscle MEPs in the right anterior tibial muscle occurred (Fig. 5 upper); concurrently, a drop in D-wave amplitude of approximately $40 \%$ was noted (Fig. 5 lower). The procedure was terminated. Some residual tumor tissue lining the cavity remained. The patient awoke from anesthesia with a monoplegia of the right leg, as had been expected based on the MEP data. Her recovery began on the 1st postoperative day, with slight movements of the toes. After 1 week, she had regained antigravity muscle strength.

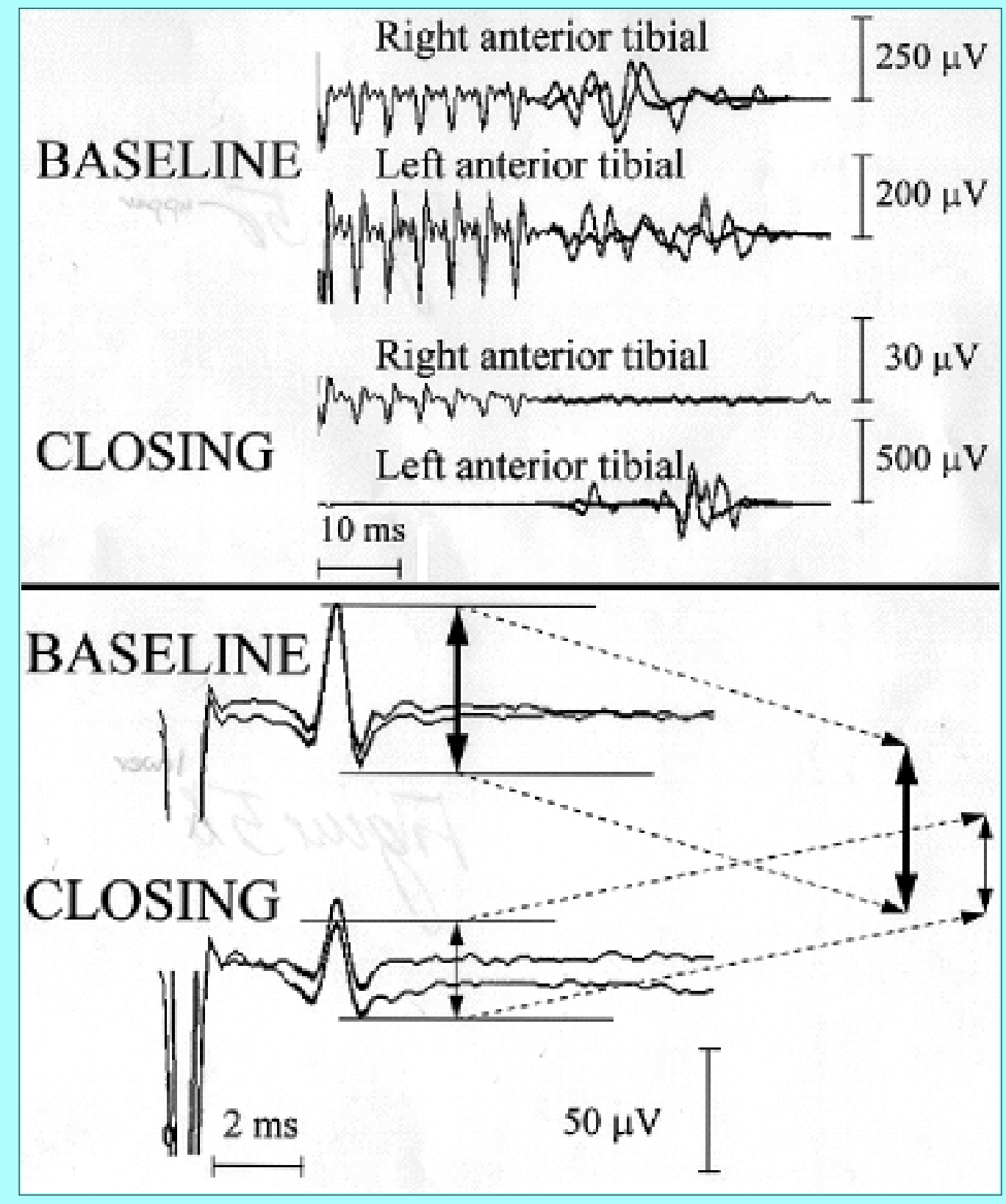

Fig. 5. Case 2. Upper: Muscle MEPs in the right leg were lost toward the end of the 
resection. Postoperatively, a transient right leg monoplegia was present. Lower: Epidural MEPs showed a decrease of the peak-to-peak D-wave amplitude of less than $50 \%$ of baseline value.

\section{Case 3}

This 9-year-old boy underwent gross-total resection of a pilocytic astrocytoma of the thoracic spinal cord that spanned four spinal segments. Preoperatively there were no motor deficits. During surgery, the muscle MEPs were lost (Fig. 6 upper) and the epidural MEP amplitude decreased, although not to less than $50 \%$ of the baseline value (Fig. 6 lower). Postoperatively the patient was paraplegic. Within 1 week he regained antigravity force in both legs, and by 2 weeks he walked again.

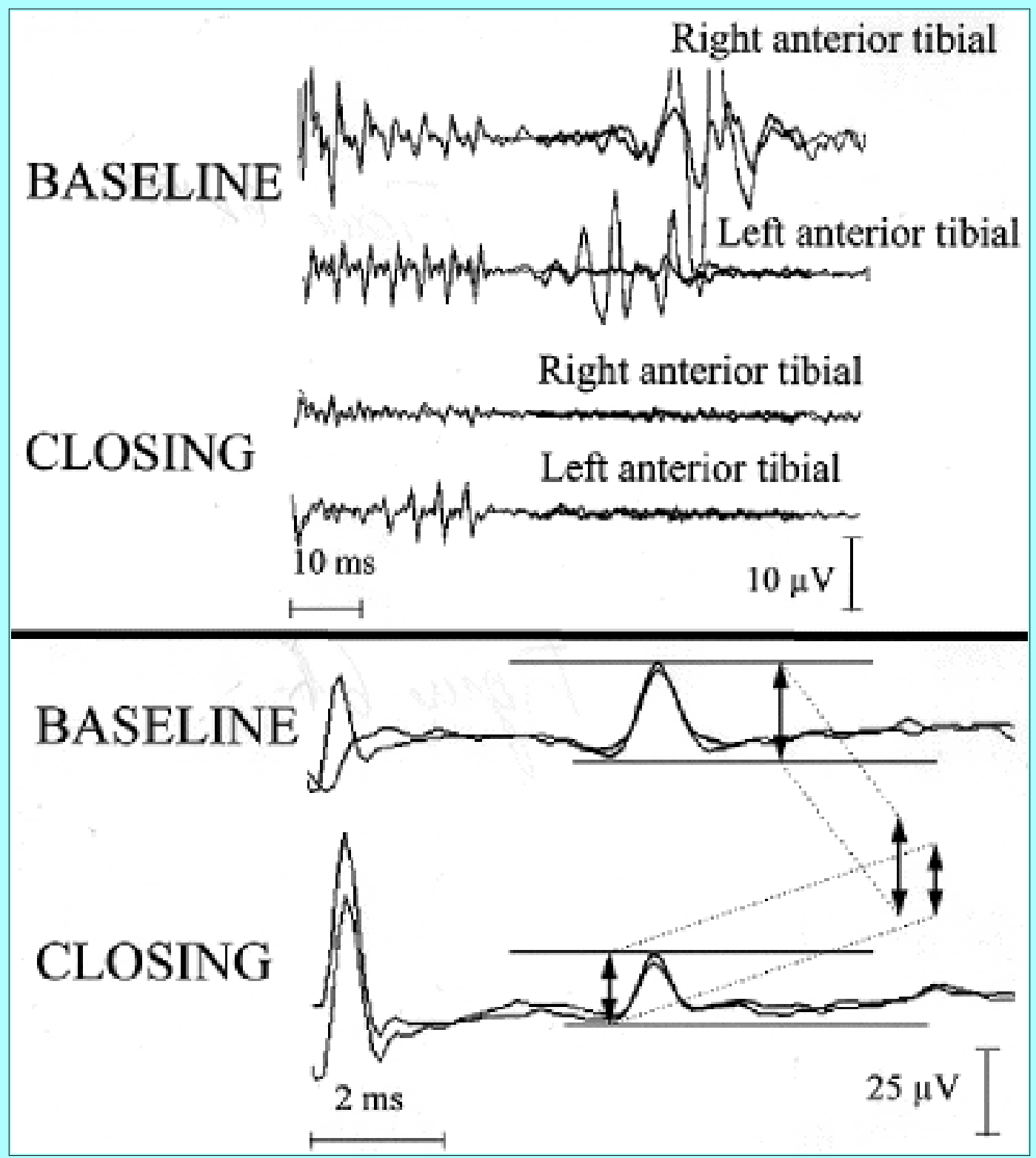

Fig. 6. Case 3. Upper: Muscle MEPs were lost in both legs during tumor resection. Lower: The D-wave amplitude declined but not by more than $50 \%$ of baseline value. The patient was paraplegic after surgery but recovered within a few days.

\section{Case 4}

This 27-year-old woman underwent resection of a C5-T1 ependymoma. Preoperatively she had slight leg 
weakness. At baseline, muscle MEPs were present in the right leg only (Fig 7 upper). The absence of a muscle response in the other leg indicated subclinical damage to the functional integrity of the motor tracts. Early in the dissection, it became clear that the tumor had extremely thinned the surrounding surviving cord tissue. However, because the epidural as well as the single-side muscle MEP recordings were stable, we were encouraged to attempt tumor removal. The tumor was then entirely resected; the D-wave amplitude decreased but remained above the 50\% limit (Fig. 7 lower). Eliciting the right leg muscle MEPs required a higher intensity and seven instead of five stimuli per train; nevertheless they remained present (Fig. 7 upper). The postoperative clinical status was not significantly changed.

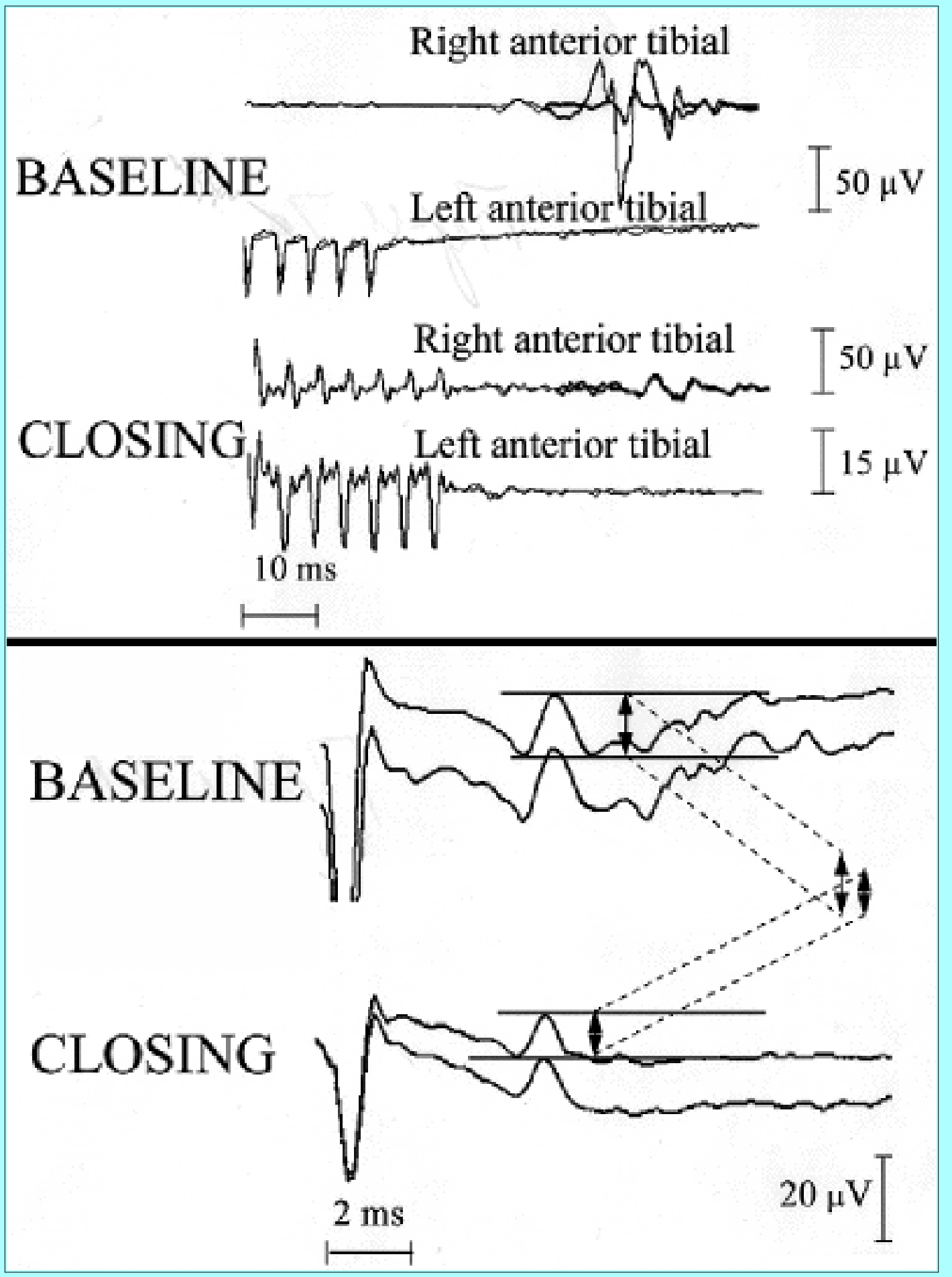

Fig. 7. Case 4. Upper: Muscle MEPs were only present on one side at the onset of the procedure. The response was preserved until the tumor was completely resected. Lower: 
D-wave amplitude declined throughout the critical part of the procedure but not by more than $50 \%$ of baseline value. The patient had no signficantly increased motor deficit.

\section{DISCUSSION}

\section{MEP Methodology}

The concept of combined epidural and muscle MEP monitoring, as used in this series, is the result of a long period of development. The present report is based on previous work done by our group, [7,8,25] and it is the first report combining intraoperative data from muscle and epidural MEPs and comparing them to the motor status in patients undergoing surgery for intramedullary spinal cord tumors.

After Merton and Morton[24] first described transcranial electrical stimulation of the motor cortex in humans in 1980, neurosurgeons quickly recognized the potential of this method to intraoperatively assess the motor pathways.[23] However, the methodological difficulties of adequate stimulation, recording, and maintenance of surgical anesthesia were formidable. Early reports of intraoperative muscle recordings in which either electrical[35] or magnetic[9] transcranial single-stimulus paradigms were used turned out to be difficult to reproduce. Based on a background of earlier experimental investigation of the motor system, the technique for recording traveling waves from the spinal cord (D-waves[20,26]) evoked by electrical motor cortex stimulation was developed for intraoperative use in humans.[2,5,7,17,33] These responses are now recorded with epidural electrodes inserted by the surgeon from the surgical field.

In Germany, the recording of muscle MEPs was significantly advanced in 1993 when Taniguchi, et al.,[31] showed that brief high-frequency trains of electrical stimuli on the exposed motor cortex reliably evoke muscle responses. Each individual electrical stimulus on the motor cortex, either with exposed cortex or with transcranial stimulation,[18] elicits a D-wave in the corticospinal tract. A fast train of five stimuli at 4-msec interstimulus intervals elicits five D-waves that travel down the corticospinal tract 4 msec apart. The spinal alpha-motor neurons are thus hit by five consecutive descending volleys, and their membrane potential is elevated above firing threshold. This mechanism has been shown with direct intracellular recordings from alpha-motor neurons in experiments in primate animals.[28] A more detailed discussion of the underlying neurophysiological principles goes beyond the scope of the present paper and is therefore subject to a separate report. Since the publication of the work by Taniguchi, et al., encouraging preliminary data[6,15,27,29] and general experience[ 8,21$]$ in using train-stimulus techniques for muscle MEP monitoring have been reported.

The parameter monitored in epidural recordings was the D-wave peak-to-peak amplitude (Fig. 5 lower). It has been shown that a decrease by more than $50 \%$ of the baseline value was associated with a long-term motor deficit.[25] Latency changes of the D-wave are rare and due to nonsurgical influences such as temperature.[7] A change in stimulus intensity also alters the D-wave latency: higher intensities lead to shorter latencies, implying that the corticospinal tract fiber activation occurs deeper in the white matter of the brain.[5]

The parameter monitored in muscle MEP recording was the presence or absence of responses. This all-or-none concept has been adopted for two reasons. 1) In contrast to epidural MEPs that show little amplitude variation,[4] the variability of muscle MEP amplitudes is tremendous.[15,22,34] Thus, defining a threshold amplitude below which one expects an intraoperative injury would be extremely difficult. 2) Our early experience as well as an analysis of the available reports (even with widely varying 
stimulus patterns) indicated that a motor deficit occurred only when the muscle response was lost.[15,22,35] The results of this series confirm this presumption.

Our data do not confirm a recent suggestion to use an elevation of thresholds to elicit muscle MEPs as a monitoring parameter.[6] The threshold for eliciting epidural MEPs did not fluctuate. On the contrary, thresholds to elicit muscle MEPs tend to vary considerably during an individual procedure (data not shown), and only presence or absence of muscle responses consistently correlated with the clinical findings. This all-or-none concept was confirmed by the present series for the anterior tibial and thenar muscles. It is not known if this concept can be applied to other muscle groups as well because their corticospinal innervation patterns are different.[13]

\section{Safety in MEP Monitoring}

Aside from direct neural tissue damage,[31] the main safety concern in the use of transcranial electrical multipulse stimulation has been the issue of seizures. No patient included in this series experienced an epileptic event.

There are no reports in the literature concerning intraoperative seizure induction with transcranial electrical stimulation in which either a single- or a train-stimulation technique was used. The term "kindling" has been indiscriminately used in this context. Kindling, however, is an experimental model referring to the induction of self-perpetuating epileptic foci in experimental animals. It requires daily, repeated electrical stimulation with $50 \mathrm{~Hz}$ for several seconds, a model that is different from the MEP train-stimulation paradigm of $250 \mathrm{~Hz}$ for $25 \mathrm{msec}$ (Fig. 2). In addition, kindling of an epileptic focus requires a long period of time (weeks to months), particularly in primates,[10] and it has not been shown to occur in humans. Furthermore, the energy necessary to induce a seizure in electroconvulsive therapy (also with 50-Hz stimulation applied for several seconds) is two orders of magnitude higher than the overall energy used for MEP monitoring.[1]

All data reported so far, including the present series as well as the theoretical concept of transcranial electrical stimulation with a short high-frequency train to elicit muscle MEPs, indicate an extremely low risk of inducing seizures. The only adverse events that occurred in this series were minor laceration and hematoma of the tongue in two patients. This is now avoided by using a padded Guedel tube to ensure proper tongue protection.

\section{Anesthesia Management}

The use of propofol for anesthesia with MEP monitoring has been reported with various stimulation techniques.[12,14,16,30,32] It is our experience that this drug together with narcotic agents is required when reliable MEP monitoring is attempted. The fact that no muscle relaxants are used, however, has been subject to considerable criticism in many discussions. Many neurosurgeons are still very reluctant to accept even the possibility of slight movement during dissection.

Why do we favor avoiding muscle relaxation? With the patient fully relaxed, muscle MEP monitoring is impossible. Others have used methods of controlled relaxation.[22] Our argument against controlled relaxation is that it would add an uncontrollable variable in interpretation of MEP data reducing the specificity of muscle MEP monitoring. On the other hand, it is unlikely that patient movement from stimulation could be completely avoided. Our experience has been that muscle relaxation was unnecessary once both surgeon and anesthesiologist became used to working without it. In a majority of patients with intramedullary tumors no twitching in the surgical field occurred. Frequently there was 
noticeable twitching, and sometimes twitching of the paraspinal muscles disturbed dissection. In such cases, continuous monitoring with muscle MEPs was impossible. It was restricted to brief breaks in microsurgical dissection. Continuous monitoring was then performed with epidural MEPs only because the single-stimulus paradigm does not elicit twitches. Thus, the two MEP techniques could still be used together. Although we are aware that this may be a controversial issue for some time, we are convinced that the benefits of using both epidural and muscle MEPs by far outweigh the inconvenience of either twitching or checking muscle responses only intermittently.

\section{Clinical Correlation of Intraoperative MEP Data}

Two parameters of epidural MEPs have been shown to have predictive value: the ability to monitor the D-wave and the intraoperative significant decline of its amplitude.[25] Patients in whom the baseline recording of epidural MEPs showed no response have a higher risk of postoperative motor deficits than those with a recordable D-wave. Whether this is due to inherent subclinical damage and "vulnerability" of the motor tract or to the fact that there was no monitoring support for the surgery is not known.

The intraoperative amplitude decrease of D-waves correlated with postoperative outcomes. If the D-wave was unchanged, the patients had no postoperative deficit. If it disappeared or declined by more than 50\% of the baseline value, the patients were permanently paraplegic, as has been shown in an earlier report.[25] Since the time of the earlier report, epidural MEP recording technology has been improved. Stimulus paradigms involving lateral stimulus points $(\mathrm{C} 1, \mathrm{C} 2, \mathrm{C} 3$, and $\mathrm{C} 4)$ have been utilized. This resulted in smaller stimulus artifacts and thus improved the quality of recordings.

Additionally, the muscle MEP recording technique was developed and applied as its value became rapidly clear. Since the time of our first report,[25] it has become evident that muscle MEPs can be preserved during surgery even if a D-wave amplitude decreases by up to $50 \%$. We suggest one possible explanation for this finding: the generation of muscle MEPs by the segmental spinal motor neuronal pool depends not only on the excitatory input from the corticospinal tract but also on the input from other descending pathways, as well as the propriospinal system. These noncorticospinal influences form a supportive system for muscle MEP generation. Thus, when the D-wave amplitude declines to some extent (30-50\%), the supportive system can compensate for that partial loss of input from the corticospinal tract. However, a greater D-wave amplitude decrease (50\%) cannot be compensated for because a critical amount of fast-conducting corticospinal tract fibers appears to be essential for both the generation of muscle MEPs and voluntary motor control. On the other hand, if the muscle MEPs disappear without a simultaneous decrease in D-wave amplitude, the supportive systems are predominantly affected by surgery. We believe that the propriospinal system, due to its diffuse nature and thus its potential for functional reorganization and plasticity, plays an essential role in both generation of muscle MEPs and postoperative motor recovery. The finding of consistent motor recovery in the patients in this series supports this concept.

In the present series it was possible to monitor epidural MEPs in two-thirds of all cases, which is approximately the same as in our previous series.[25] The D-waves are generated by the corticospinal tract axons; therefore, conus medullaris tumors cannot be monitored with epidural MEPs. In the other patients with no tumor in the conus medullaris, absence of D-waves was associated with previous spinal cord radiotherapy or due to extensive tumor compression. Occasionally, scarring from a previous surgery prohibited insertion of a recording electrode.

Muscle MEPs were essentially monitorable in all patients. In some cases, only one leg response could be 
obtained. Preoperatively, in patients with a paralyzed extremity, no muscle MEPs were ever recordable. Thus, preoperative motor status was adequately reflected by intraoperative baseline MEP recordings. In those patients in whom no epidural MEPs could be monitored, muscle MEPs alone were utilized. The intraoperative changes of both epidural and muscle MEPs reflected optimal sensitivity for significant impairment of the functional integrity of the corticospinal tract. All postoperative deficits were detected by MEP monitoring. The specificity of changes was $91 \%$. Together with a $100 \%$ ability to monitor patients without significant preoperative motor deficits, this represents a technique that is applicable in all spinal cord tumor operations.

The practical influence of MEP data on the course of surgery either reassured that motor function would be intact or warned that the functional integrity of the motor pathways was jeopardized. In all cases in the present series, MEP monitoring was considered an essential part of the procedure, constantly assuring the surgeon that the surgical manipulations were not too injurious. Because mere documentation of irreversible injury would be an insufficient role for intraoperative monitoring to play, the major advantage of this combined muscle and epidural MEP monitoring was the warning it gave before permanent injury occurred. From the earlier experience, it was clear that a loss of D-wave predicted permanent motor deficit.[2,25]

With improved D-wave recording quality the incremental decrease in D-wave amplitude became better discernible. Thus the surgeon had a window of warning about damage to the motor system before this damage became irreversible. Muscle MEP recording was a significant practical improvement because it provided more information. As long as muscle MEPs were present, the absence of a motor deficit was certain, even if the D-wave amplitude began to decline. A loss of muscle MEPs then indicated serious damage to the motor system but was reversible as long as the D-wave amplitude did not decline by more than 50\%. In this situation the epidural MEP amplitude again became the only parameter to follow if, for surgical reasons, the procedure could not be terminated.

Most spinal cord tumors are microsurgically removed in a piecemeal fashion. Thus if MEPs deteriorate, the surgery can be stopped before gross-total resection is achieved. After clinical recovery, a second surgery can be considered, particularly in patients with ependymoma in whom complete tumor resection is essential for long-term progression-free survival. In some situations, however, when an en-bloc removal is necessary, as for instance in patients with hemangioblastomas, this warning is still only of documentary value. This, however, is a limitation imposed by the anatomical nature of the lesion rather than a shortcoming of the monitoring technique.

All postoperative motor deficits that occurred in our patients were transient. Thus MEP monitoring warned the surgeon of imminent danger to the functional integrity of the motor tract. In our earlier series of patients undergoing surgical treatment of intramedullary tumors, two patients with permanent postoperative paraplegia were described: in one of them the D-wave was lost and in the other there was a greater than 50\% amplitude reduction.[25] This occurred at a time when muscle MEPs elicited with the train-stimulation technique were not available.

\section{Further Validation of MEP Monitoring}

To achieve increased scientific acceptance for the role of MEPs in intraoperative patient care, a prospective controlled trial would be necessary. In the course of such a clinical trial a patient group would have to serve as control, thus undergoing operation without monitoring or without communication of monitoring data to the surgeon. We do not consider it ethically acceptable for our own practice to 
undertake such a trial on a single-institution basis. The difficulties of a multicenter trial involving different surgeons with different levels of experience as well as different monitoring personnel with varying levels of experience are formidable. Under these circumstances and given the relatively small incidence of spinal cord tumors, as well as the limited number of centers performing a sufficient number of procedures each year, a prospective controlled trial is unlikely to be feasible in the near future, and it is arguably unethical to attempt one.

\section{CONCLUSIONS}

The combined intraoperative monitoring of epidural and muscle MEPs elicited by transcranial electrical stimulation used in this consecutive series of 100 surgically treated intramedullary spinal cord tumors correctly corresponded to the motor clinical findings. The presence of muscle MEPs always indicated intact motor function. Intraoperative loss of muscle MEPs indicated a temporary loss of motor function in the corresponding limb as long as epidural MEP amplitude did not decline below $50 \%$ of the baseline value. Further decline of the amplitude could indicate permanent paraplegia or, in the case of a high cervical tumor, quadriplegia.

The technique used for evoking motor potentials is safe. It can be readily implemented in a routine neurosurgical environment. It identifies impairment of the functional integrity of the motor pathways and could be considered a warning sign before a permanent deficit results.

\section{Acknowledgments}

The authors are indebted to San-San Chiang, Linda Velazquez, Ingrid Kothbauer-Margreiter, Matevz Krzan, Nobu Morota, Shlomo Constantini, and Stephen J. Thompson.

\section{References}

1. Barker AT, Freeston IL, Jalinous R, et al: Magnetic and electrical stimulation of the brain: safety aspects, in Rossini PM, Marsden CD (eds): Non-Invasive Stimulation of Brain and Spinal Cord: Fundamentals and Clinical Applications. Neurology and Biology, Vol 41. New York: Alan R. Liss, 1988, pp 131-144

2. Boyd SG, Rothwell JC, Cowan JMA, et al: A method of monitoring function in corticospinal pathways during scoliosis surgery with a note on motor conduction velocities. J Neurol Neurosurg Psychiatry 49:251-257, 1986

3. Burke D, Hicks R, Stephen J, et al: Assessment of corticospinal and somatosensory conduction simultaneously during scoliosis surgery. Electroencephalogr Clin Neurophysiol 85:388-396, 1992

4. Burke D, Hicks R, Stephen J, et al: Trial-to-trial variability of corticospinal volleys in human subjects. Electroencephalogr Clin Neurophysiol 97:231-237, 1995

5. Burke D, Hicks RG, Stephen JPH: Corticospinal volleys evoked by anodal and cathodal stimulation of the human motor cortex. J Physiol (Lond) 425:283-299, 1990

6. Calancie B, Harris W, Broton JG, et al: "Threshold-level" multipulse transcranial electrical stimulation of motor cortex for intraoperative monitoring of spinal motor tracts: description of method and comparison to somatosensory evoked potential monitoring. J Neurosurg 88:457-470, 1998 
7. Deletis V: Intraoperative monitoring of the functional integrity of the motor pathways. Adv Neurol 63:201-214, 1993

8. Deletis V, Kothbauer K: Intraoperative neurophysiology of the corticospinal tract, in Stålberg E, Sharma HS, Olsson Y (eds): Spinal Cord Monitoring. Vienna: Springer-Verlag, (In press, 1998)

9. Edmonds HL Jr, Paloheimo MPJ, Backman MH, et al: Transcranial magnetic motor evoked potentials (tcMMEP) for functional monitoring of motor pathways during scoliosis surgery. Spine 14:683-686, 1989

10. Engel J Jr: Seizures and Epilepsy. Philadelphia: FA Davis, 1989, pp 80-84

11. Epstein FJ, Farmer JP: Pediatric spinal cord tumor surgery. Neurosurg Clin North Am 1:569-590, 1990

12. Fennelly ME, Taylor BA, Hetreed M: Anaesthesia and the motor evoked potential, in Jones SJ, Boyd S, Hetreed M, et al. (eds): Handbook of Spinal Cord Monitoring. Dordrecht: Kluwer, 1994, pp $272-276$

13. Jankowska E, Padel Y, Tanaka R: Projections of pyramidal tract cells to alpha-motoneurones innervating hind-limb muscles in the monkey. J Physiol (Lond) 249:637-667, 1975

14. Jellinek D, Jewkes D, Symon L: Noninvasive intraoperative monitoring of motor evoked potentials under propofol anesthesia: effects of spinal surgery on the amplitude and latency of motor evoked potentials. Neurosurgery 29:551-557, 1991

15. Jones SJ, Harrison R, Koh KF, et al: Motor evoked potential monitoring during spinal surgery: responses of distal limb muscles to transcranial cortical stimulation with pulse trains.

Electroencephalogr Clin Neurophysiol 100:375-383, 1996

16. Kalkman CJ, Drummond JC, Ribberink AA, et al: Effects of propofol, etomidate, midazolam and fentanyl on motor evoked responses to transcranial electrical or magnetic stimulation in humans.

Anesthesiology 76:502-509, 1992

17. Katayama Y, Tsubokawa T, Maejima S, et al: Corticospinal direct response in humans: identification of the motor cortex during intracranial surgery under general anaesthesia. J Neurol Neurosurg Psychiatry 51:5059, 1988

18. Katayama Y, Tsubokawa T, Yamamoto T, et al: Spinal cord potentials to direct stimulation of the exposed motor cortex in humans: comparison with data from transcranial motor cortex stimulation, in Rossini PM, Marsden CD (eds): Non-Invasive Stimulation of Brain and Spinal Cord: Fundamentals and Clinical Applications. Neurology and Biology, Vol 41. New York: Alan R. Liss, 1988, pp 305-311

19. Kernell D, Chien-Ping W: Post-synaptic effects of cortical stimulation on forelimb motoneurones in the baboon. J Physiol (Lond) 191:673-690, 1967

20. Kernell D, Chien-Ping WU: Responses of the pyramidal tract to stimulation of the baboon's motor cortex. J Physiol (Lond) 191:653-672, 1967

21. Kothbauer K, Deletis V, Epstein FJ: Intraoperative spinal cord monitoring for intramedullary surgery: an essential adjunct. Pediatr Neurosurg 26:247-254, 1997 
22. Lang EW, Beutler AS, Chestnut RM, et al: Myogenic motor-evoked potential monitoring using partial neuromuscular blockade in surgery of the spine. Spine 21:1676-1686, 1996

23. Levy WJ, York DH, McCaffrey M, et al: Motor evoked potentials from transcranial stimulation of the motor cortex in humans. Neurosurgery 15:287-302, 1984

24. Merton PA, Morton HB: Stimulation of the cerebral cortex in the intact human subject. Nature 285:227, 1980

25. Morota N, Deletis V, Constantini S, et al: The role of motor evoked potentials during surgery for intramedullary spinal cord tumors. Neurosurgery 41:1327-1336, 1997

26. Patton HD, Amassian VE: Single-and multiple unit analysis of cortical stage of pyrimidal tract activation. J Neurophysiol 17:345-363, 1954

27. Pechstein U, Cedzich C, Nadstawek J, et al: Transcranial high-frequency repetitve electrical stimulation for recording myogenic motor evoked potentials with the patient under general anesthesia.

Neurosurgery 39:335-344, 1996

28. Phillips CG, Porter R: The pyramidal projection to motoneurones of some muscle groups of the baboon's forelimb. Progr Brain Res 12:222-245, 1964

29. Rodi Z, Deletis V, Morota N, et al: Motor evoked potentials during brain surgery. Pflüger's Archiv-Eur J Physiol 431:R291-292, 1996

30. Schmid UD, Boll J, Liechti S, et al: Influence of some anesthetic agents on muscle responses to transcranial magnetic cortex stimulation: a pilot study in humans. Neurosurgery 30:85-92, 1992

31. Taniguchi M, Cedzich C, Schramm J: Modification of cortical stimulation for motor evoked potentials under general anesthesia: technical description. Neurosurgery 32:219-226, 1993

32. Taniguchi M, Nadstawek J, Langenbach U, et al: Effects of four intravenous anesthetic agents on motor evoked potentials elicited by magnetic transcranial stimulation. Neurosurgery 33:407-415, 1993

33. Thompson PD, Day BL, Crockard HA, et al: Intra-operative recording of motor tract potentials at the cervico-medullary junction following scalp electrical and magnetic stimulation of the motor cortex. J Neurol Neurosurg Psychiatry 54:618-623, 1991

34. Woodforth IJ, Hicks RG, Crawford MR, et al: Variability of motor-evoked potentials recorded during nitrous oxide anesthesia from the tibialis anterior muscle after transcranial electrical stimulation. Anesth Analg 82:744-749, 1996

35. Zentner J: Noninvasive motor evoked potential monitoring during neurosurgical operations in the spinal cord. Neurosurgery 24:709-712, 1989

Manuscript received March 18, 1998.

Accepted in final form April 30, 1998.

Some of the data in this article were included in presentations at the American Association of 
Neurological Surgeons annual meeting, Philadelphia, PA, April 25-30, 1998 (Paper \#761), and at the 7th International Symposium on Spinal Cord Monitoring, Osaka, Japan, March 18-20, 1998.

Address reprint requests to: Karl Kothbauer, M.D., Institute of Neurology and Neurosurgery, Beth Israel Medical Center North Division, 170 East End Avenue, New York, New York 10128. e-mail: kkothbau@bethisraelny.org. 